\title{
RESEARCH
}

Open Access

\section{Co-culture of hWJMSCs and pACs in double biomimetic ACECM oriented scaffold enhances mechanical properties and accelerates articular cartilage regeneration in a caprine model}

Yu Zhang ${ }^{1,2,3,4}$, Chunxiang Hao ${ }^{5}$, Weimin Guo ${ }^{1,2,3}$, Xiaoyu Peng ${ }^{6}$, Mingjie Wang ${ }^{1,2,3}$, Zhen Yang ${ }^{7}$, Xu Li $^{7}$, Xueliang Zhang ${ }^{8}$, Mingxue Chen ${ }^{1,2,3}$, Xiang Sui ${ }^{1,2,3}$, Jiang Peng ${ }^{1,2,3}$, Shibi Lu ${ }^{1,2,3}$, Shuyun Liu ${ }^{1,2,3^{*}}$, Quanyi Guo ${ }^{1,2,3^{*}}$ and Qing Jiang ${ }^{4^{*}}$

\begin{abstract}
Background: The dedifferentiation of chondrocytes and the unstable chondrogenic differentiation status of pluripotent mesenchymal stem cells (MSCs) are immense issues in cell-based articular cartilage repair and regenerative strategies. Here, to improve the cartilage characteristics of seed cells, a double biomimetic acellular cartilage extracellular matrix (ACECM)-oriented scaffold was used to mimic the cartilage microenvironment for human umbilical cord Wharton's jelly-derived MSCs (hWJMSCs) and primary cartilage cells (pACs) to regenerate hyaline cartilage.
\end{abstract}

Methods: A double biomimetic ACECM-oriented scaffold was created from the cartilage extracellular matrix of pig articular cartilage using pulverization decellularization freeze-drying procedures. hWJMSCs and pACs were cocultured at ratios of 50:50 (co-culture group, ACCC), 0:100 (ACAC group) and 100:0 (ACWJ group) in the ACECMoriented scaffold, and the co-culture system was implanted in a caprine model for 6 months or 9 months to repair full-thickness articular cartilage defects. The control groups, which had no cells, comprised the blank control (BC) group and the ACECM-oriented scaffold (AC) group. Gross morphology and magnetic resonance imaging (MRI) as well as histological and biomechanical evaluations were used to characterize the cartilage of the repair area.

\footnotetext{
*Correspondence: clear_ann@163.com; doctorguo_301@163.com; qingj@nju.edu.cn

'Institute of Orthopaedics, Chinese PLA General Hospital, 28 Fuxing Road, Haidian District, Beijing 100853, China

${ }^{4}$ Department of Sports Medicine and Adult Reconstructive Surgery, Nanjing Drum Tower Hospital, The Affiliated Hospital of Nanjing University Medical School, 321 Zhongshan Road, Gulou District, Nanjing 210008, China Full list of author information is available at the end of the article
}

(c) The Author(s). 2020 Open Access This article is licensed under a Creative Commons Attribution 4.0 International License, which permits use, sharing, adaptation, distribution and reproduction in any medium or format, as long as you give appropriate credit to the original author(s) and the source, provide a link to the Creative Commons licence, and indicate if changes were made. The images or other third party material in this article are included in the article's Creative Commons licence, unless indicated otherwise in a credit line to the material. If material is not included in the article's Creative Commons licence and your intended use is not permitted by statutory regulation or exceeds the permitted use, you will need to obtain permission directly from the copyright holder. To view a copy of this licence, visit http://creativecommons.org/licenses/by/4.0/ The Creative Commons Public Domain Dedication waiver (http://creativecommons.org/publicdomain/zero/1.0/) applies to the data made available in this article, unless otherwise stated in a credit line to the data. 
(Continued from previous page)

Results: Relative to the control groups, both the gross morphology and histological staining results demonstrated that the neotissue of the ACCC group was more similar to native cartilage and better integrated with the surrounding tissue. Measurements of glycosaminoglycan content and Young's modulus showed that the repair areas had more abundant cartilage-specific content and significantly higher mechanical strength in the ACCC group than in the control groups, especially at 9 months. On MRI, the T2-weighted signal of the repair area was homogeneous, and the oedema signal disappeared almost completely in the ACCC group at 9 months. HLA-ABC immunofluorescence staining demonstrated that hWJMSCS participated in the repair and regeneration of articular cartilage and escaped surveillance and clearance by the caprine immune system.

Conclusion: The structure and components of double biomimetic ACECM-oriented scaffolds provided a cartilagelike microenvironment for co-cultured seed cells and enhanced the biomechanics and compositions of neotissue. This co-culture system has the potential to overcome the dedifferentiation of passage chondrocytes and the unstable chondrogenic differentiation status of MSCs.

Keywords: Acellular cartilage extracellular matrix-oriented scaffold, Human umbilical cord Wharton's jelly-derived mesenchymal stem cell, Primary cartilage cell, Co-culture system

\section{Background}

Articular cartilage injury, usually caused by engaging in exercise, professional training and games, trauma due to accident, or being overweight, has become increasingly common among young populations and professional athletes. A retrospective study of 5233 knee arthroscopies by Lukasik et al. reported that over $57 \%$ of patients were diagnosed with a cartilage lesion [1]. In addition, the overall prevalence of articular cartilage injury in athletes $(36 \%)$ is more than twice the rate in the general population [2]. However, cartilage rarely self-heals because it lacks vascular and lymph tissue to provide nutrients [3]; thus, osteoarthritis generally develops within 520 years $[4,5]$. Cell-based treatment strategies are promising methods to repair and regenerate articular cartilage and to stop the progression of osteoarthritis $[6,7]$.

However, the histological characteristics of neotissue are usually highly inferior to those of native articular cartilage mainly because of the unstable chondrogenic differentiation status of mesenchymal stem cells (MSCs), which results in the presence of hypertrophic or calcified chondrocytes [8-11]. In addition, for autologous chondrocyte implantation $(\mathrm{ACI})$ or matrix -induced chondrocyte implantation (MACI), chondrocytes also face some problems, including limited cartilage source to isolate primary articular cartilage cells (pACs) $[8,12]$ and dedifferentiation after several passages [13]. In recent decades, it has been emphasized that the environment plays a critical role in the regulation of cell behaviour [14-16]. Co-culture has been explored to promote the chondrogenic induction of MSCs and to maintain the native phenotype of chondrocytes by bio-stimulation via cell-cell interactions in coculture systems $[14,17,18]$. In addition, co-culture reduces the required amount of cartilage tissue, decreases the in vitro culture time and lowers the degree of chondrocyte degeneration [13]. However, some studies showed that co-culture merely promoted a unidirectional trophic effect of MSCs on chondrocytes [19, 20]. These differences are probably attributable to the complex microenvironment, which (i) comprises a milieu of biochemical, biomechanical and bioelectrical signals derived from surrounding cells, the extracellular matrix (ECM) and soluble factors and (ii) regulates cell metabolism and function.

In vitro, a co-culture system was constructed using unpassaged pACs possessing their native phenotype and an ACECM-oriented scaffold retaining native cartilage tissue components and oriented structures [21-23]; hWJMSCs were displayed as an alternative cells source for cartilage tissue engineering because of distinct chondrogenic potential, highly proliferative activity and safety for patients $[24,25]$. In vitro, hWJMSCs were cocultured with chondrocytes and successfully differentiated into chondrocytes, as shown by the significant upregulation of collagen II and aggrecan genes [26]. In gross morphology, engineered tissue was translucent and resilient, similar to hyaline cartilage tissue. Despite these findings, the regeneration and repair conditions after the implantation of engineered tissue into cartilage defects in vivo remain unknown. Here, we evaluated whether the co-culture of hWJMSCs and pACs in a double biomimetic ACECM-oriented scaffold could achieve a joint surface that mimics the native state as closely as possible and return the articular cartilage to its natural biomechanics and compositions.

\section{Materials and methods \\ Preparation of seed cells and construction of tissue- engineered cartilage in vitro}

The collection of three healthy human umbilical cords for this study was approved by the Ethics Committee of the General Hospital of the People's Liberation Army (PLA), and written informed consent was provided by all 
mothers. The detailed protocols for the isolation and culture of hWJMSCs and pACs have been previously described [26, 27]. According to previously described protocols, hWJMSCs were isolated using micromass mesenchymal tissue adhesion on a culture dish containing Dulbecco's modified Eagle's medium/-Ham's F 12 (Sigma, USA) nutrient medium (DMEM/F12) (containing $10 \%$ foetal bovine serum (FBS) (Corning, USA), 100 $\mathrm{mg} / \mathrm{ml}$ penicillin, $10 \mathrm{mg} / \mathrm{ml}$ streptomycin and $250 \mathrm{mg} / \mathrm{ml}$ amphotericin B). At passage 3, a subset of the isolates was prepared for co-culture with pACs. pACs were isolated from slices of goat shoulder articular cartilage by cutting them into microscopic-pieces and digesting them using $0.15 \%$ type II collagenase for $2 \mathrm{~h}$ at $37^{\circ} \mathrm{C}$. Then, the individual cells and cell sheet were collected by centrifugation at $250 \times g$ for $5 \mathrm{~min}$ at room temperature. The cells were resuspended in DMEM/F12 culture medium supplemented with $10 \%$ FBS and antibiotics $(100 \mathrm{mg} / \mathrm{ml}$ penicillin, $10 \mathrm{mg} / \mathrm{ml}$ streptomycin and $250 \mathrm{mg} / \mathrm{ml}$ amphotericin B) and cultured in $25-\mathrm{cm}^{2}$ culture flasks with $5 \% \mathrm{CO}_{2}$ in a $37^{\circ} \mathrm{C}$ incubator. The pACs were collected for construction of the co-culture system at approximately $80 \%$ confluence.

A three-dimensional (3D)-oriented scaffold was made from acellular ECM using the freeze-drying technique previously described [21]. After cross-linking by dehydrothermal and water-soluble carbodiimide treatment, oriented scaffolds with a diameter of $6 \mathrm{~mm}$ and a thickness of $1 \mathrm{~mm}$ were produced and sterilized by ${ }^{60} \mathrm{Co}-\gamma$ irradiation (at $5 \mathrm{mrad}$ ). A total of $10^{7} \mathrm{pACs}$ and hWJMSCs were mixed in three different ratios: 100:0, 0: 100 and 50:50 in $100 \mu \mathrm{l}$ DMEM/F12 medium. Then, the cell suspension was seeded into a 3D-oriented scaffold and incubated for $2 \mathrm{~h}$ at $37^{\circ} \mathrm{C}$ with $5 \% \mathrm{CO}_{2}$ to promote cell adhesion to the scaffolds. Next, cell-scaffold complexes were transferred to 6-well cell culture plates, and $5 \mathrm{ml}$ of culture medium (DMEM/F12, 10\% FBS, $100 \mathrm{mg} /$ $\mathrm{ml}$ penicillin, $10 \mathrm{mg} / \mathrm{ml}$ streptomycin and $250 \mathrm{mg} / \mathrm{ml}$ amphotericin B) was added. Tissue-engineered cartilage was acquired after cultivation for 3 weeks at $37^{\circ} \mathrm{C}$ under $5 \% \mathrm{CO}_{2}$. The gross morphology was evaluated according to size, colour and shape.

\section{Cell viability and distribution assessment in the 3D- oriented scaffolds}

Dead/live staining was used to analyse cell viability after culturing in the scaffolds for 3 days. Briefly, cell-scaffold complexes were stained with FDA (fluorescein diacetate, $5 \mu \mathrm{g} / \mathrm{ml}$, Sigma) and PI (propidium iodide, $5 \mu \mathrm{g} / \mathrm{ml}$, Sigma) for $5 \mathrm{~min}$ and rinsed 3 times with PBS (phosphate-buffered solution, $\mathrm{pH}$ 7.4). The samples were immediately visualized using a laser scanning confocal microscope (Olympus FV 1200, Japan) [26]. Red and green fluorescence indicate dead and live cells, respectively.
To observe the distribution of seed cells in the 3D-oriented scaffold, scanning electron microscopy (SEM) was applied to view the samples in the sagittal plane and in cross sections after culturing for 3 weeks. All samples were fixed in 2.5\% glutaraldehyde, dehydrated in a series of graded ethanol to $100 \%$ ethanol, treated with hexamethyldisilazane and sputtercoated with gold-palladium before observation [21].

\section{In vivo experiments using the engineered tissue}

To verify the effectiveness and safety of engineered tissue, the ACCC (pACs: hWJMSCs, 50:50) group was used to repair full-thickness articular cartilage defects of the femoral condyles in a caprine model. The animal experimental procedures followed the standards for the care and use of laboratory animals and were approved by the Institutional Animal Care and Use Committee of the Chinese PLA General Hospital. Three healthy goats were randomly assigned to each of the following groups per time point: a blank control group (BC, full-thickness articular cartilage defects without any treatment), an ACECM-oriented scaffold group (AC, treatment with ACECM-oriented scaffolds), a pACs+ACECMoriented scaffold group (ACAC, treatment with pACs and ACECM-oriented scaffolds), a hWJMSCs+ACECM-oriented scaffold group (ACWJ, treatment with hWJMSCs and ACECM-oriented scaffolds) and a ACCC group (ACCC, treatment with hWJMSCs, pACs and ACECM-oriented scaffolds). Goats (white, male, $30 \pm 5 \mathrm{~kg}$ ) were treated with sumianxin II $(0.1 \mathrm{~mL} / \mathrm{kg})$ and ketamine $(4 \mathrm{mg} / \mathrm{kg})$ by intramuscular injection. After location in the supine position on operating table, sterilizing knee joint and spreading the drapes, the medical parapatellar longitudinal skin incision is $8-10-\mathrm{cm}$ long. The capsule of the knee is exposed, and longitudinal-shaped capsulotomy is performed. Lateral dislocation of the patella and knee flexion at $120^{\circ}$ was performed, and then the medical and lateral femoral condyles are exposed. Full-thickness articular cartilage defects in femoral condyles were made using a 6.5-mm-diameter corneal trephine under sterile conditions, while preserving the subchondral bone. Penicillin sodium was administered for 3 days to prevent infection. The goats were allowed to move freely in a comfortable environment after surgery.

\subsection{T high-resolution magnetic resonance imaging evaluation}

At 6 or 9 months after surgery, all goats were euthanized by intravenous pentobarbital overdose. MRI images of the entire fresh knee joints were obtained immediately using a volume coil on a laboratory 7.0 T MRI scanner (Bruker Biospec, Germany). T2-weighted spin-echo images with fat suppression were obtained in the sagittal, frontal and transverse planes. The imaging parameters were as follows: slice thickness $(1.0 \mathrm{~mm})$, echo time $(36 \mathrm{~ms})$, repetition time $(5109 \mathrm{~ms})$, flip angle $\left(90^{\circ}\right)$, acquisition time $(18 \mathrm{~min})$ and field of view $(75 \times 75 \mathrm{~mm})$. All images were scored 
independently according to the International Cartilage Repair Society (ICRS) Whole-Organ MRI Score (WORMS) of the knee in OA by an experienced radiologist specialized in musculoskeletal medicine and blinded to the group assignments [28].

\section{Gross morphology and scoring}

After MRI, the whole knee joints, including the femoral condyles, the corresponding meniscus and tibia plateau, and the surrounding synovial membrane, were exposed, photographed and grossly evaluated. The repair tissues were examined macroscopically and scored by three blinded observers using the ICRS scoring system for cartilage repair, including the degree of repair, integration, surface regularity and overall judgement [29].

\section{Histological assessment and scoring}

Cartilage repair areas were fixed in $4 \%$ buffered formalin for 1 week, and decalcified in 10\% EDTA for 4 weeks. After dehydration, repair tissues were embedded in paraffin and sectioned into $8-\mu \mathrm{m}$ slices. Histology sections were stained with haematoxylin and eosin (H\&E) and safranin "O" [27]. Three experienced pathologists scored the repair tissue in a blinded manner using the ICRS scoring system, which includes cartilage thickness, surface regularity, integration with adjacent host cartilage, cell morphology and matrix staining [30].

\section{Immunohistochemical staining}

After deparaffinization, rehydration and antigen retrieval using boiled citric acid, tissue slices were incubated in primary antibodies for collagen type I (ab23446, Abcam, England) and collagen type II (ab34712, Abcam, England) at $4{ }^{\circ} \mathrm{C}$ overnight. Then, the primary antibody was washed away with PBS, and a MaxVision ${ }^{\mathrm{Ta}}$ Detection System DAB kit (MaiXin Bio, China) was used to measure the colour according to the manufacturer's protocol.

\section{Nanoindentation test and glycosaminoglycan analyses}

To assess the biomechanical properties of the repair tissue, Young's elastic modulus was measured using the In Situ Nanomechanical Test System (Hysitron, USA) in medium at room temperature. The radius of curvature of the cospherical diamond probe tip was $100 \mu \mathrm{m}$. The trapezoidal load function was used on each indent site with loading $(10 \mathrm{~s})$, holding $(5 \mathrm{~s})$ and unloading $(10 \mathrm{~s})$. The cylindrical loading device was positioned perpendicular to the repair area and advanced forward by $2000 \mathrm{~nm}$ at $200 \mathrm{~nm} / \mathrm{s}$.

The repair tissue was immediately extracted by the corneal trephine. The samples were crushed, and Grocott's Methenamine Silver (GMS) Kit (Genmed Scientifics Inc., USA) was used to test the glycosaminoglycan (GAG) content according to manufacturer's instructions. The GAG content ( $\mu \mathrm{g} / \mathrm{sample})$ was calculated as a component of the histological analysis.

\section{HLA-ABC immunofluorescence staining}

To determine whether hWJMSCs participated in the repair and regeneration of articular cartilage, a cell tracking technique was applied via HLA-ABC immunofluorescence staining. Briefly, the slices were dewaxed and dehydrated and the antigen was retrieved. Then, the slices were placed in 1/100 HLA-ABC primary antibody (DCABH-9508, Abcam, England) at $4{ }^{\circ} \mathrm{C}$ overnight. After washing away the primary antibody, the slices were incubated for $1 \mathrm{~h}$ with green fluorescent secondary antibody and for $5 \mathrm{~min}$ with Hoechst 33258 nuclear stain, and images were captured using an Olympus fluorescence microscope.

\section{Statistical analysis}

Data were analysed using SPSS 18.0 (SPSS Inc., Chicago, IL, USA). The quantified values are reported as the means \pm standard deviation. One-way analysis of variance (ANOVA) with the Student-Newman-Keuls (SNK) test was used to determine significant differences between the two groups, $n=3$. A value of $P<0.05$ was considered to indicate a significant difference.

\section{Results}

Gross morphology of the tissue-engineered cartilage, cell viability and distribution in the 3D-oriented scaffold

After co-culture in ACECM-oriented scaffolds for 3 weeks in vitro, gross morphology characteristics of the tissue-engineered cartilage, including appearance, size and colour, were evaluated. In the cell-scaffold groups (including the ACAC, ACWJ and ACCC groups), the pores were almost filled with cells and ECM, and the size of the constructs was larger than that of the AC group. The constructs in the cell-scaffold groups appeared glossier and more semi-transparent than those in the AC group, and they had an appearance similar to native cartilage tissue (Fig. 1a).

After seeding into scaffolds for 3 days, cell viability was assessed using dead/live staining. Confocal microscopy showed that green fluorescent cells (living cells) were spread out in the 3D-oriented scaffold and were the main component in each group, even though some red fluorescent cells (dead cells) were dispersed in the scaffold. Thus, the 3D ACECM-oriented scaffold did not display significant cytotoxicity (Fig. 1b).

To explore the microstructure of the cell-scaffold, SEM was used to analyse the distribution of cells and the extracellular topological structure in the scaffold. The scaffold exhibited a honeycomb structure on cross-sections and stacked compartments on the sagittal plane. Among the cell-scaffold groups, the ACWJ group showed a high cell density, while the cells were sparse in the ACAC group. In 


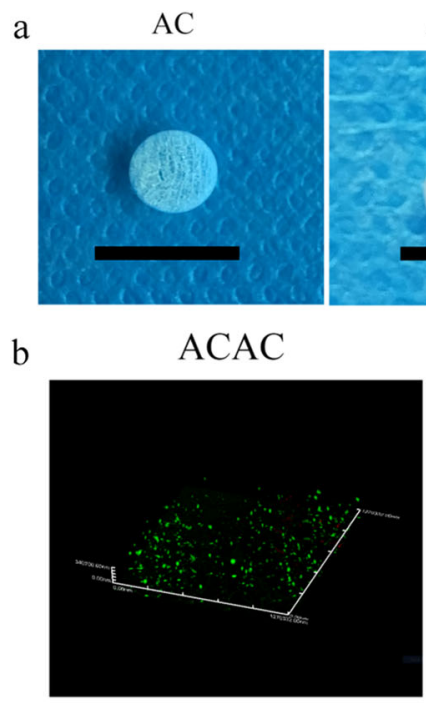

c
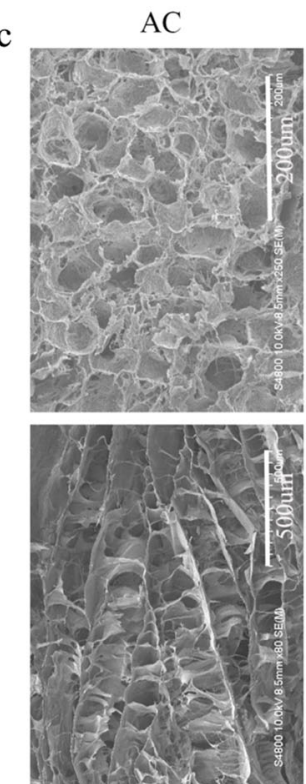

ACAC
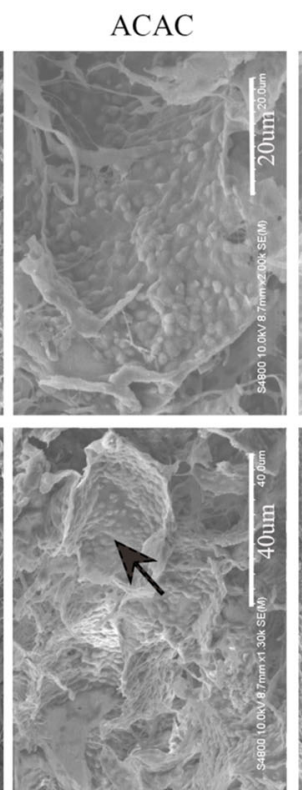

ACWJ

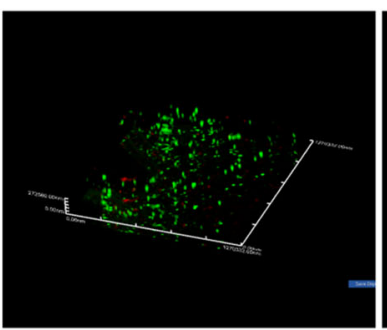

ACWJ
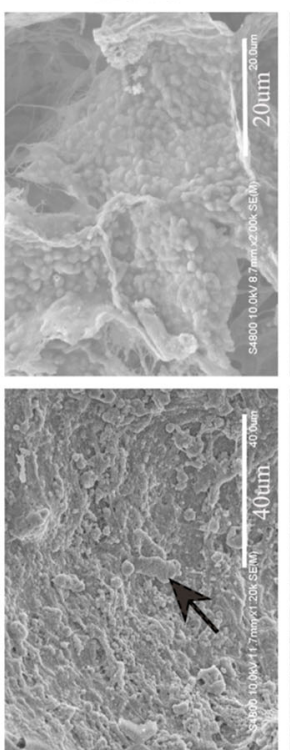

ACCC

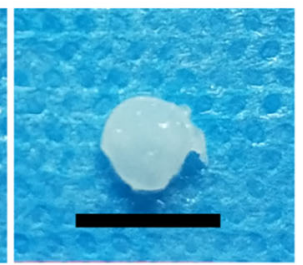

$\mathrm{ACCC}$

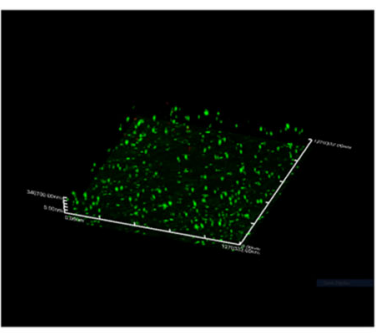

ACCC
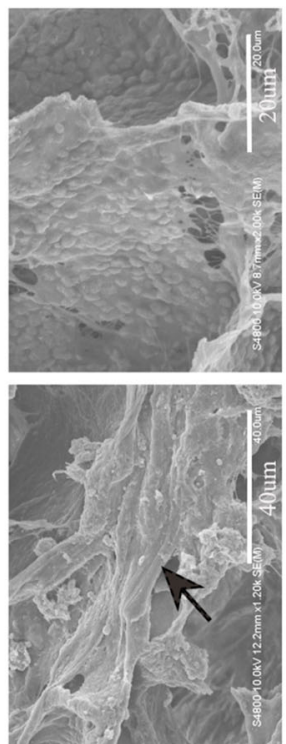

Fig. 1 Hyaline cartilage-like gross morphology and directional distribution of cells and ECM after co-culture in ACECM-oriented scaffold. a After culture for 3 weeks, the 3D ACECM-oriented scaffolds were filled with cells and extracellular matrix in the ACAC, ACWJ and ACCC groups, and the appearance of the engineered tissue was semitransparent and glossy. The scale of black bar is $1 \mathrm{~cm}$. $\mathbf{b}$ Dead/live staining under 3D confocal fluorescence microscopy. The 3D-oriented scaffolds are mainly filled with green fluorescent cells (living cells), with just a few red fluorescence cells (dead cells) present after cultivation for 3 days. c SEM results. The upper row shows the high-power (HP) field, and the lower row shows the low-power (LP) field; AC group, the upper and lower pictures are cross-sectional and sagittal plane views, respectively. In the ACAC, ACWJ and ACCC groups, the HP field shows cells distributed in holes, and the LP field shows the topological structure of the ECM; black arrows indicate the topological microstructure of ECM secreted by cells

view of the topological microstructure of ECM secreted by cells, the ACCC group presented thick and continuous connectivity among pores, while the ACWJ and ACAC groups had short and slim morphology, which is critical to guarantee strong mechanical properties, such as pressure and shear forces (Fig. 1c).

\section{In vivo experiments}

One goat died during surgery because of an anaesthetic overdose, and another goat's knee (in the $\mathrm{ACWJ}$ group) became infected on the 8th day after the operation but was later cured. Excluding the cases of infection and death, the remaining goats were included in the results analysis.

Neotissue in the ACCC group with mature cartilage MRI signal and complete subchondral bone

MRI of the fresh whole knee joints of the ACCC group at 6 months showed that the damaged joints were almost completely filled with neotissue, and their surfaces were smooth. However, some cavities were still found in the 
repair area in the ACAC group and the ACWJ group, and obvious defects were still present in the AC group and the $\mathrm{BC}$ group. At 9 months, the neotissue was uniform and similar to that of native cartilage, and the oedema signal of subchondral bone had completely disappeared. The defects in the ACWJ group, the AC group and the ACAC group were filled with neotissue, but the MRI results showed that some joint effusion or immature cartilage signal existed (Fig. 2a).

Regarding the WORMS results of the knee, the cartilage signal and morphology scores were better in the ACWJ group (6 months, $2.0 \pm 1.0$; 9 months, $1.3 \pm 0.6$ ) and the ACCC group (6 months, $1.3 \pm 1.5$; 9 months, $1.6 \pm 0.6$ ) than in the $\mathrm{AC}$ group or the $\mathrm{BC}$ group. The ACAC group exhibited improved neotissue quality at 9 months compared with that at 6 months. With respect to subchondral bone attrition, the $\mathrm{BC}$ group and the $\mathrm{AC}$ group were markedly concave $(5.3 \pm 0.6 ; 6.3 \pm 1.2)$ and slightly concave $(2.8 \pm 0.7 ; 1.7 \pm 0.4)$ at 6 months and 9 months, respectively. Compared with the other groups, the marrow abnormality scores were lowest in the ACCC group, with < $25 \%$ abnormal regions. The total WORMS results revealed the following: the best neotissue quality and repair was found in the knees from the ACCC group and the ACWJ group, followed by the ACAC group and the AC group. The $\mathrm{BC}$ group had the worst WORMS results, with no any repair or regeneration observed (Fig. 2b).

\section{The ACCC group achieved faster repair and higher ICRS- MECR scores than the other groups}

At 6 months, the cartilage defects were not completely repaired with neotissue in any group. However, the ACCC group was the only group in which a full-thickness cartilage defect was not observed. At 9 months, the repair area was plump and glossy because of the regeneration of neotissue in the ACCC group and the ACWJ group, but a local area of partial-thickness defects intermixed with areas of normal thickness still existed in the $\mathrm{AC}$ group and the $\mathrm{ACAC}$ group. The $\mathrm{BC}$ group showed almost no repair, or the depth of the defects was quite large (Fig. 3a).

The ICRS-MECR results showed that the repair and regeneration in the ACCC group (6 months, $10.0 \pm 1.0$; 9 months, $11.0 \pm 1.7$ ) was significantly better than that in the ACAC group (6 months, $7.0 \pm 2.0$; 9 months, $9.7 \pm$ 0.6 ), the AC group (6 months, $4.7 \pm 1.2$; 9 months, $7.3 \pm$ 2.1 ) and the $\mathrm{BC}$ group (6 months, $1.1 \pm 0.4$; 9 months, $1.5 \pm 0.7$ ), which highlights the intense integration with the border zone, the large degree of defect repair and the hyaline macroscopic appearance (Fig. 3b).

\section{Histological level revealed abundant hyaline cartilage components in the ACCC group}

The H\&E results showed that at 6 months, neotissue padded the defects and covered and tightly aligned with the subchondral bone, and the thickness of the neotissue was similar to that of the surrounding cartilage in the ACCC group, whereas in the $\mathrm{BC}$ group, the subchondral bone was naked and concave. The neotissue in the AC group was thinner and less evenly distributed than in the cell-scaffold groups, including the ACAC group, the $\mathrm{ACWJ}$ group and the ACCC group. At 9 months, a distribution of mixed columnar clusters, a smooth surface and normal subchondral bone could be clearly observed in the ACCC group. The cartilage in the AC group did not integrate with the surrounding normal cartilage, and the cartilage in the ACAC group had an uneven surface (Fig. 4a).

Safranin "O" staining revealed that the ECM of neotissue had a sharp outline and showed different thicknesses and various grades of maturity. At 6 months, the ACWJ group and the $\mathrm{AC}$ group showed immature neotissue that appeared as loose fibrocartilage-like tissue. Compared with the ACCC group, other groups showed infrequent cartilage lacuna formation and dense matrix masses in the repair area at 9 months (Fig. 4b).

Immunohistochemical staining at 6 months showed that the ACCC group was negative for collagen I whereas the $\mathrm{AC}$ group and the $\mathrm{ACWJ}$ group were not. And collagen I at 9 months in the ACCC group was slightly higher than AC group. The ACCC group was significantly positive for collagen II at both 6 months and 9 months, while the control groups but the AC group showed negative staining results. Compared with the AC group, collagen II was less expressed in the ACCC group at 6 months, but there was no significant difference at 9 months (Fig. $4 \mathrm{c}, \mathrm{d}$ ).

According to the ICRS-VHAS, the ACCC group (6 months, $13.3 \pm 1.2$; 9 months, $14.7 \pm 2.1$ ), the ACAC group (6 months, $11.7 \pm 1.5$; 9 months, $12.7 \pm 1.2$ ) and the ACWJ group (6 months, $11.7 \pm 1.5$; 9 months, $13.0 \pm$ 2.0) achieved better tissue repair than the $\mathrm{BC}$ group (6 months, $0.7 \pm 0.6 ; 9$ months, $1.0 \pm 1.0$ ). The significant advantages of the ACCC group over the other cellscaffold complex groups included the formation of mature hyaline cartilage-like ECM and a columnar or clustered cell distribution (Fig. 4e).

\section{Biomechanical and glycosaminoglycan quantitative analyses}

As a critical functional indicator of articular cartilage, Young's modulus was accurately measured using the nanoindentation technique. At 6 months, the Young's modulus of the ACCC group $(3.9 \pm 0.9)$ was significantly higher than those of the other groups, including the ACWJ group (2.2 \pm 0.6$)$, the ACAC group $(2.1 \pm 0.4)$ and the AC group $(1.7 \pm 0.3)$. The difference between the ACCC group and cell-scaffold groups increased at 9 months (Fig. 5a). 


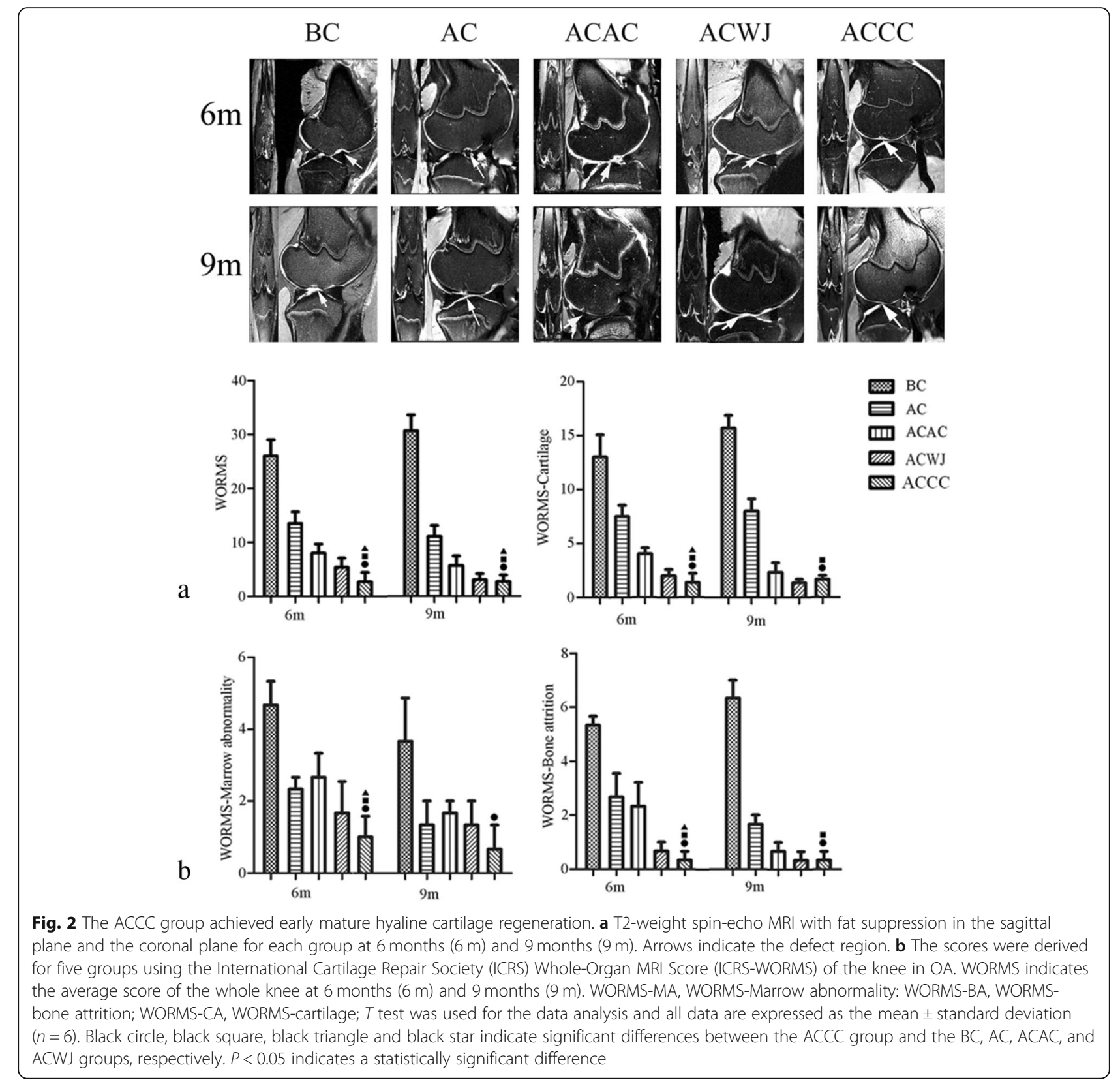

The total GAG content per sample showed no significant difference among the cell-scaffold groups, but it was more abundant in the ACCC group at 6 months. The GAG content of the ACCC group was higher than that of the $\mathrm{AC}$ group at 9 months, while no significant difference was found between the ACAC group and the ACWJ group (Fig. 5b).

\section{hWJMSCs participated in the repair and regeneration of} cartilage defects

As a human leukocyte antigen I molecular marker, HLA$\mathrm{ABC}$ immunofluorescence staining can provide evidence demonstrating that hWJMSCs are one part of the neotissue and evade from surveillance of the immune system. Green fluorescence can be observed in the repair area of the ACCC group, while it is absent in the normal area, indicating that hWJMSCs were present in the repair area and were an important component of neotissue (Fig. 6).

\section{Discussion}

A co-culture system provides a potential strategy for the repair and regeneration of cartilage

Articular cartilage injury is a common sports-related disease that severely affects subsequent achievements and career planning. A co-culture system provides a win-win strategy to eliminate issues related to chondrocytes and 


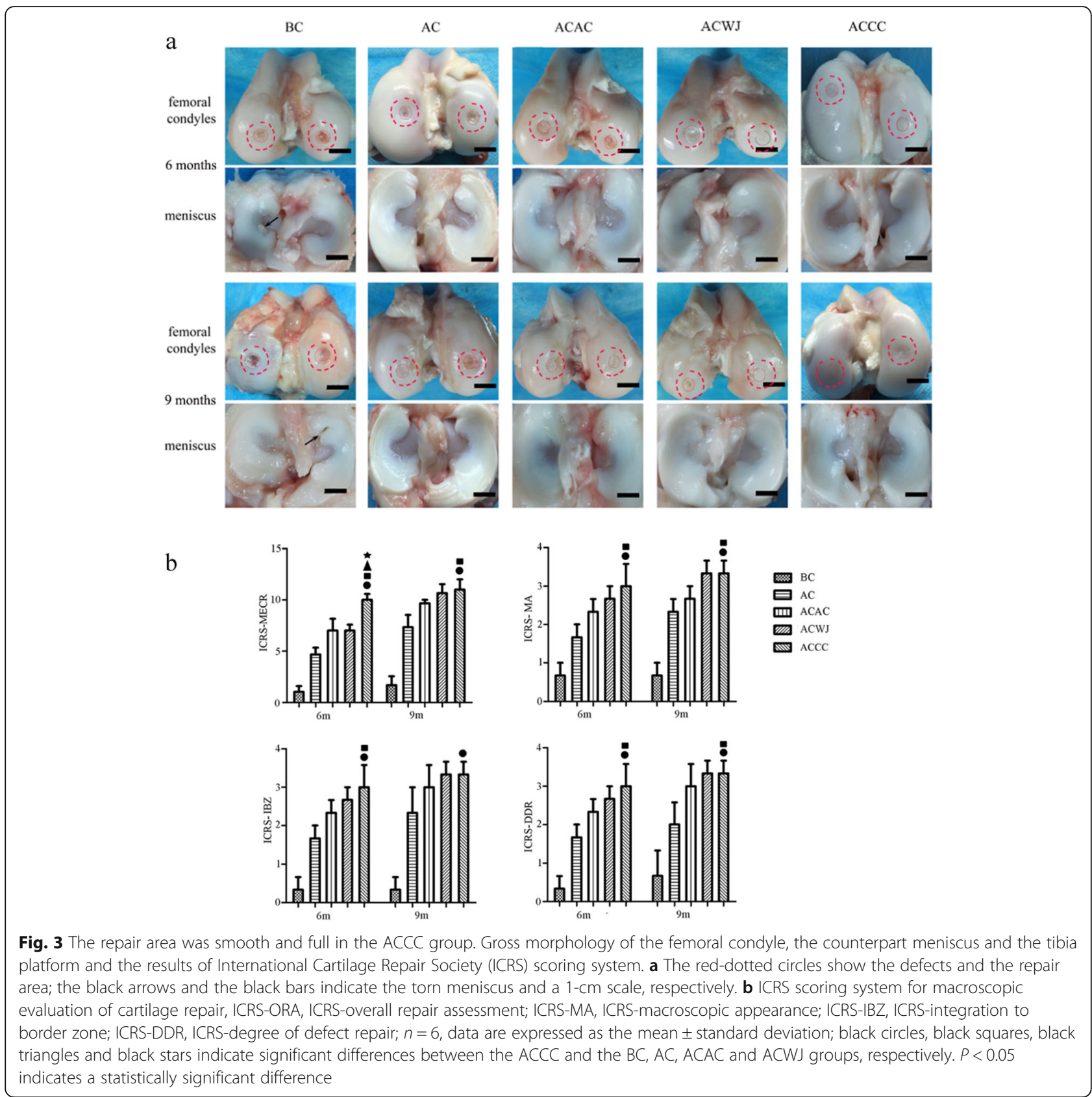

MSCs and facilitate high-quality cartilage regeneration. The results obtained in vivo indicated the following: MRI showed that there was no significant joint effusion or bone marrow oedema signal in the ACCC group, and the neotissue of the ACCC group showed a smooth surface and uniform signal. Gross morphology showed that the ACCC group successfully achieved complete repair and regeneration at 6 months, and the histological properties of the neotissue were highly similar to those of native articular cartilage, including the thickness of the neotissue, the columnar cell distribution, the formation of cartilage lacuna and the high expression of collagen
II. Although the damaged cavities observed in the ACWJ group were also completely covered with neotissue at 6 months, both the Young's modulus and GAG content per sample in the ACCC group were improved at 6 months and especially at 9 months, highlighting the superiority of the regeneration in this group. From these animal experiments, we conclude that the group implanted with only the ACECM-oriented scaffold, without any seed cells, required much more time for neotissue generation to occur. The HLA-ABC measurements also provided important data that indirectly confirmed that the hWJMSCs possessed low immunogenicity and escaped destruction by 


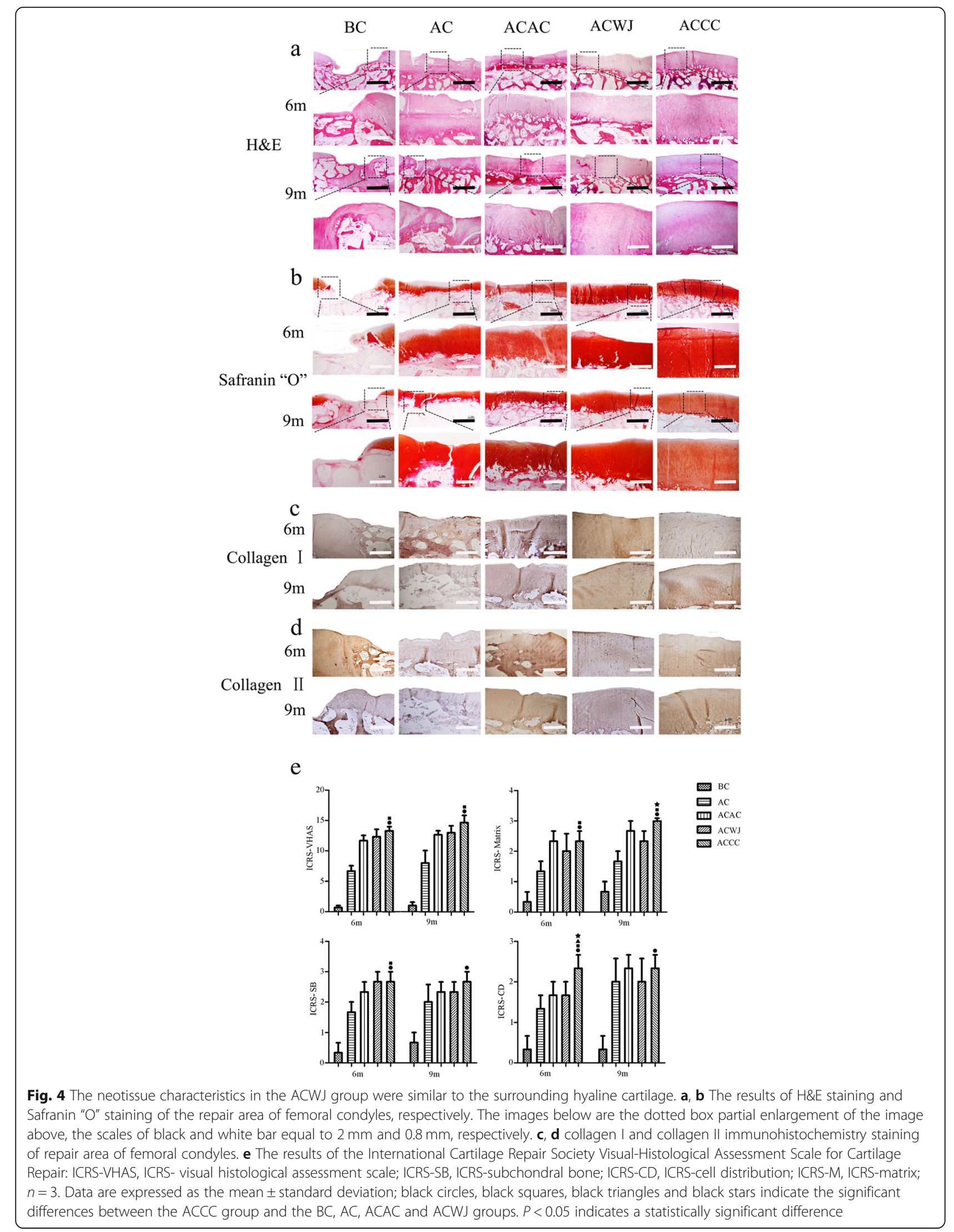



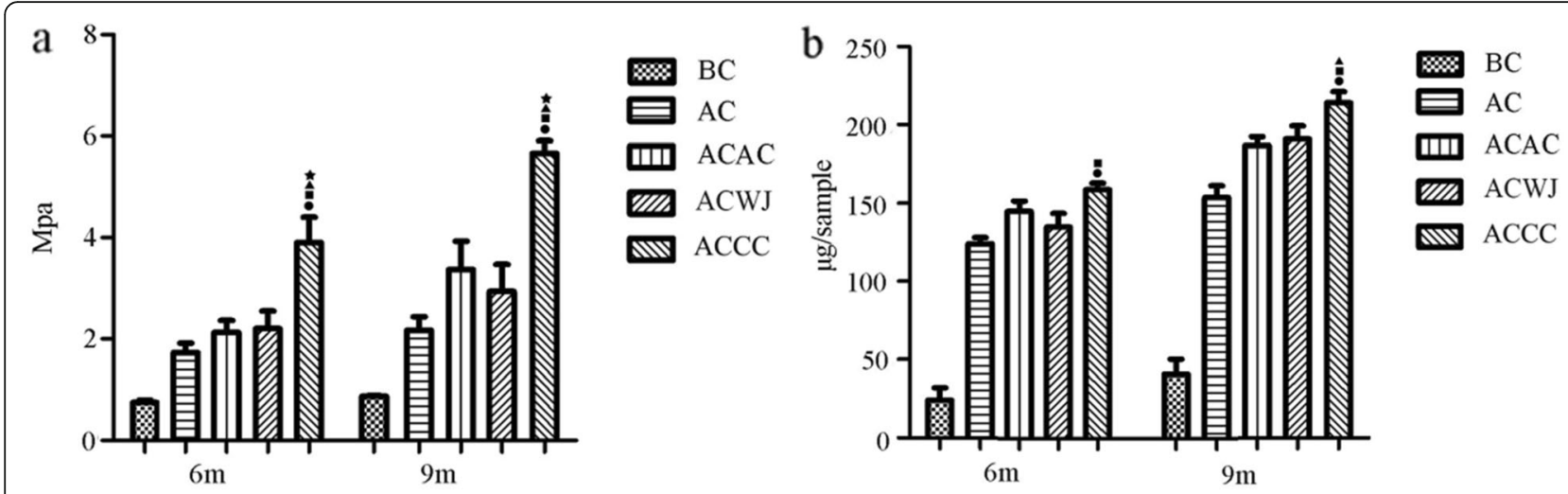

Fig. 5 The biochemical and biomechanical characteristics of the ACCC group were significantly better than those of the other control groups. Quantitative analysis of articular cartilage repair. Biochemical and biomechanical evaluation was performed to quantify a Young's modulus and $\mathbf{b}$ the glycosaminoglycan (GAG) content in the three samples. A $T$ test was used for data analysis, and all data are expressed as the mean \pm standard deviation, with $n=6$. Black circles, black squares, black triangles and black stars indicate the significant differences between the ACCC group and the BC, AC, ACAC, and ACWJ groups. $P<0.05$ indicates a statistically significant difference

the immune system. This result is congruent with previous findings showing that hWJMSCs elicit a very low immune response [31-33].

\section{The biological microenvironment affects cellular} behaviour and represents a potential repair mechanism elicited by engineered cartilage tissue

In this paper, engineered cartilage tissue was successfully constructed by culturing hWJMSCs in a simulated cartilage microenvironment (consisting of pACs and ACECMoriented scaffolds), and this construct facilitated the repair and regeneration of full-thickness defects of the femoral condyle in a caprine model. The potential mechanisms may involve (i) the primitive hyaline cartilage phenotype and the improved activity of pACs or (ii) the stable chondrogenic differentiation of hWJMSCs because of mild and durable regulation of the biomimetic cartilage microenvironment, not the temporary and unique action of a chondrogenic inducer. The in vitro results from our previous study revealed that hWJMSCs in a co-culture system were successfully induced into chondrocytes without significant upregulation of collagen X [26]. Other researchers have also reported

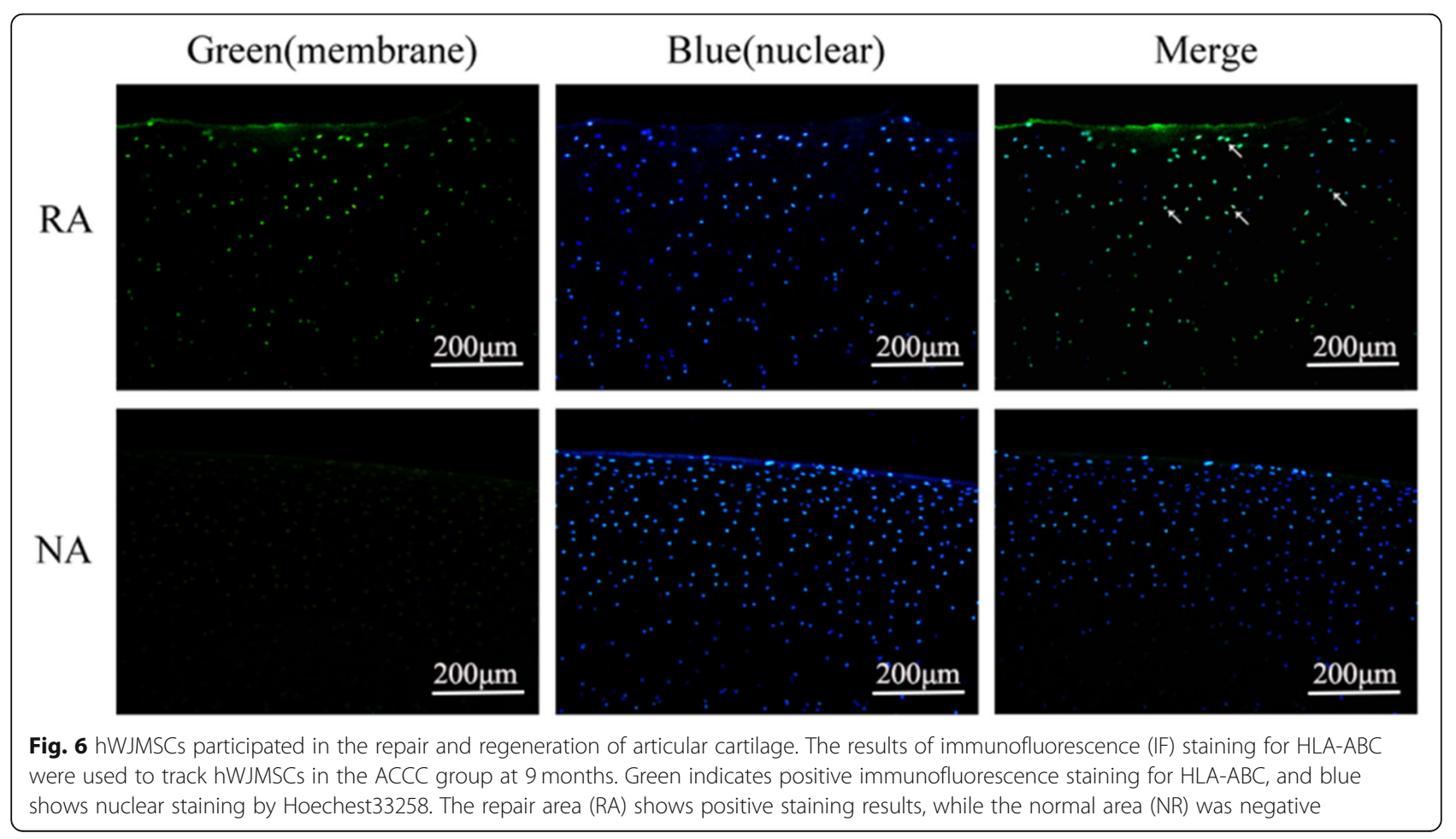


that hWJMSCs can inhibit hypertrophy and improve chondrogenesis, as articular chondrocytes secrete parathyroid hormone-related protein (PTHrP) in co-culture systems $[11,34]$. In addition, hWJMSCs possess better chondrogenic differentiation capacity than bone marrow mesenchymal stem cells (BMSCs) [24, 35, 36]. These factors may contribute greatly to chondrogenic differentiation and the regeneration of articular cartilage via hWJMSCs. It was also demonstrated by other studies in vivo that co-culture of MSCs and chondrocytes possessed obviously potential to repair and regeneration of articular cartilage compared with monoculture $[37,38]$. MSCs differentiated into chondrocytes under co-culture system microenvironment adding no inducers in these two studies, which is similar to our study.

In the co-culture system, hWJMSCs rather than pACs eliminate the obstacle of limited cartilage resources and prevent the dedifferentiation of chondrocytes after expansion in vitro [39-41]. Additionally, the oriented microstructure of the scaffold can direct collagen fibre and ECM-oriented arrangement, which also plays an important role in enhancing the biomechanical strength of engineered cartilage tissue [42, 43]; however, biomechanical testing was not performed due to the short cultivation time in vitro.

\section{Limitations}

Some questions remain unanswered in this study, and further research needs to be carried out to elucidate the functional mechanisms in the co-culture system. For example, the specific cytokines secreted by pACs or hWJMSCs and their signalling pathways remain unclear; how they regulate the cellular biobehaviour is also unknown. It remains unknown what constitutes the optimal construct, including optimal cell number MSC to AC ratio and direct or indirect co-culture models. The results showed that in as long as 9 months, the longer term stability of this construct is unknown. Moreover, in this study on a large animal model, immunological rejection of hWJMSCs through the search of specific antibodies has not been investigated. The risk of spreading disease is another disadvantage for patients if hWJMSCs are derived from an allogeneic umbilical cord. These findings are very important for guiding the development and optimization of coculture models and for constructing tissue-engineered cartilage in one step without chondrocytes and the clinical application of hWJMSCs in the future. As for the experimental model, two defects were made in medical and lateral femoral condyles of one knee joint, which probably influence each other's repair results.

\section{Conclusion}

Co-culture of hWJMSCs and pACs in a spatial structure and component biomimetic ACECM-oriented scaffold showed clear advantages: pACs maintained a good primitive chondrocyte phenotype, and hWJMSCs remained in a stable chondrogenic differentiation status in the biomimetic cartilage microenvironment, which greatly contributed to achieve high-quality regeneration of injured cartilage and yielded a product that was similar to the native articular cartilage in terms of its biomechanics and tissue components. Future studies should investigate the regulation mechanisms of underlying the cellular behaviour in this co-culture system and explore the efficacy and safety of this system for future clinical applications.

\section{Abbreviations}

ACECM: Acellular cartilage extracellular matrix; BMSCs: Bone marrow mesenchymal stem cells; DMEM/F12: Dulbecco's modified Eagle's medium/Ham's F 12; FBS: Foetal bovine serum; FDA: Fluorescein diacetate;

GAG: Glycosaminoglycan; hWJMSCs: Human umbilical cord Wharton's jellyderived MSCs; ICRS: International Cartilage Repair Society; ICRS-

VHAS: International Cartilage Repair Society Visual-Histological Assessment Scale; MSCs: Mesenchymal stem cells; OCT: Optimum cutting temperature; pACs: Primary articular cartilage cells; PBS: Phosphate-buffered solution; PI: Propidium iodide; PTHrP: Parathyroid hormone-related protein; WORMS: Whole-Organ MRI Score

\section{Acknowledgements}

We acknowledge the staff working with the Bruker Biospec 7.0 T MRI scanner at the China Nanotechnology Center and the staff working with the Hysitron Tribo Indenter In Situ Nanomechanical Test System at Peking University.

\section{Authors' contributions}

QG, SL and QJ designed the study; WG, MW, XS, JP, CH and SL obtained the reagents and methodologies. $Y Z$ performed the research and wrote the paper. ZW, XL, XZ and XP performed the animal experiments and analysed the data. All authors read and approved the final manuscript.

\section{Funding}

This work was supported by the National Key R\&D Fund (2019YFA0110600), the National Natural Science Foundation of China (81772319) and the Translation Medicine Fund of the Chinese PLA General Hospital (2017TM014).

Availability of data and materials

The datasets used and/or analysed during the current study are available from the corresponding author on reasonable request.

\section{Ethics approval and consent to participate}

All animal experiments were approved by the II Local Bioethical Committee in Warsaw (approval number: $31 / 2011$ ) and performed according to the Guidelines for the Regulation of Animal Experiments.

\section{Consent for publication}

Not applicable.

\section{Competing interests}

The authors declare that they have no competing interests.

\section{Author details}

${ }^{1}$ Institute of Orthopaedics, Chinese PLA General Hospital, 28 Fuxing Road, Haidian District, Beijing 100853, China. ${ }^{2}$ Beijing Key Lab of Regenerative Medicine in Orthopaedics, 28 Fuxing Road, Haidian District, Beijing 100853, China. ${ }^{3}$ Key Laboratory of Musculoskeletal Trauma \& War Injuries, PLA, 28 Fuxing Road, Haidian District, Beijing 100853, China. ${ }^{4}$ Department of Sports Medicine and Adult Reconstructive Surgery, Nanjing Drum Tower Hospital, The Affiliated Hospital of Nanjing University Medical School, 321 Zhongshan Road, Gulou District, Nanjing 210008, China. Institute of Anesthesia, Chinese PLA General Hospital, 28 Fuxing Road, Haidian District, Beijing 100853, China. ${ }^{6}$ Department of Geriatrics, Nanjing Drum Tower Hospital, The Affiliated 
Hospital of Nanjing University Medical School, 321 Zhongshan Road, Gulou District, Nanjing 210008, China. ${ }^{7}$ School of Medicine, Naikai University, Tianjin 300071, China. ${ }^{8}$ Shanxi Traditional Chinese, No. 46 Binzhou west Street, YingZe District, Taiyuan 030001, China.

\section{Received: 28 January 2020 Revised: 20 March 2020} Accepted: 6 April 2020 Published online: 19 May 2020

\section{References}

1. Widuchowski W, Lukasik P, Kwiatkowski G, Faltus R, Szyluk K, Widuchowski J, Koczy B. Isolated full thickness chondral injuries. Prevalance and outcome of treatment. Acta Chir Orthop Traumatol Cech. 2008;75(5):382-6.

2. Flanigan DC, Harris JD, Trinh TQ, Siston RA, Brophy RH. Prevalence of chondral defects in athletes' knees: a systematic review. Med Sci Sports Exerc. 2010;42(10):1795-801.

3. Mankin HJ. The structure, chemistry and metabolism of articular cartilage. Bull Rheum Dis. 1967;17(7):447-52.

4. Chen D, Shen J, Zhao W, Wang T, Han L, Hamilton JL, Im HJ. Osteoarthritis: toward a comprehensive understanding of pathological mechanism. Bone Res. 2017;5:16044

5. Lenoch F. Disturbance of nourishment of the articular cartilage and its relation to the development of osteoarthrosis. Review Czechoslovak Med. 1970;16(3):113-7.

6. Wang M, Yuan Z, Ma N, Hao C, Guo W, Zou G, Zhang Y, Chen M, Gao S, Peng J, Wang A, Wang Y, Sui X, Xu W, Lu S, Liu S, Guo Q. Advances and prospects in stem cells for cartilage regeneration. Stem Cells Int. 2017;2017: 4130607.

7. Nazempour A, Van Wie BJ. Chondrocytes, mesenchymal stem cells, and their combination in articular cartilage regenerative medicine. Ann Biomed Eng. 2016;44(5):1325-54.

8. Vasiliadis HS, Wasiak J, Salanti G. Autologous chondrocyte implantation for the treatment of cartilage lesions of the knee: a systematic review of randomized studies. Knee Surgery Sports Traumatol Arthroscopy. 2010; 18(12):1645-55.

9. Niemeyer P, Salzmann GM, Hirschmuller A, Sudkamp NP. Factors that influence clinical outcome following autologous chondrocyte implantation for cartilage defects of the knee. Zeitschrift fur Orthopadie und Unfallchirurgie. 2012;150(1):83-8.

10. Harris JD, Siston RA, Brophy RH, Lattermann C, Carey JL, Flanigan DC. Failures, re-operations, and complications after autologous chondrocyte implantation--a systematic review. Osteoarthritis Cartilage. 2011;19(7):77991.

11. Fischer J, Dickhut A, Rickert M, Richter W. Human articular chondrocytes secrete parathyroid hormone-related protein and inhibit hypertrophy of mesenchymal stem cells in coculture during chondrogenesis. Arthritis Rheum. 2010;62(9):2696-706.

12. Ruano-Ravina A, Jato Diaz M. Autologous chondrocyte implantation: a systematic review. Osteoarthritis Cartilage. 2006;14(1):47-51.

13. Ouyang X, Wei B, Mao F, Zhang X, Xu Y, Wang L. Uncultured bone marrow mononuclear cells delay the dedifferentiation of unexpanded chondrocytes in pellet culture. Cell Tissue Res. 2015;361(3):811-21.

14. Miki $Y$, Ono K, Hata S, Suzuki T, Kumamoto $H$, Sasano $H$. The advantages of co-culture over mono cell culture in simulating in vivo environment. J Steroid Biochem Mol Biol. 2012;131(3-5):68-75.

15. Weber CE, Kuo PC. The tumor microenvironment. Surg Oncol. 2012;21(3): 172-7.

16. Zhong $L$, Huang $X$, Karperien M, Post JN. The regulatory role of signaling crosstalk in hypertrophy of MSCs and human articular chondrocytes. Int J Mol Sci. 2015;16(8):19225-47.

17. Koseska A, Bastiaens PI. Cell signaling as a cognitive process. EMBO J. 2017; 36:568-82. PMID: 28137748. https://doi.org/10.15252/embj.201695383.

18. Lawrence TS, Beers WH, Gilula NB. Transmission of hormonal stimulation by cell-to-cell communication. Nature. 1978;272(5653):501-6.

19. Wu L, Prins HJ, Helder MN, van Blitterswijk CA, Karperien M. Trophic effects of mesenchymal stem cells in chondrocyte co-cultures are independent of culture conditions and cell sources. Tissue Eng A. 2012;18(15-16):1542-51.

20. Wu L, Leijten JC, Georgi N, Post JN, van Blitterswijk CA, Karperien M. Trophic effects of mesenchymal stem cells increase chondrocyte proliferation and matrix formation. Tissue Eng A. 2011;17(9-10):1425-36.

21. Yang Q, Peng J, Guo Q, Huang J, Zhang L, Yao J, Yang F, Wang S, Xu W, Wang A, Lu S. A cartilage ECM-derived 3-D porous acellular matrix scaffold for in vivo cartilage tissue engineering with PKH26-labeled chondrogenic bone marrow-derived mesenchymal stem cells. Biomaterials. 2008;29(15): 2378-87.

22. Kang H, Peng J, Lu S, Liu S, Zhang L, Huang J, Sui X, Zhao B, Wang A, Xu W, Luo Z, Guo Q. In vivo cartilage repair using adipose-derived stem cellloaded decellularized cartilage ECM scaffolds. J Tissue Eng Regen Med. 2014;8(6):442-53.

23. Qiang $Y$, Yanhong $Z$, Jiang $P$, Shibi $L$, Quanyi G, Xinlong M, Qun X, Baoshan $X$, Bin Z, Aiyuan W, Li Z, Wengjing X, Chao Z. Xenoimplantation of an extracellular-matrix-derived, biphasic, cell-scaffold construct for repairing a large femoral-head high-load-bearing osteochondral defect in a canine model. Sci World J. 2014;2014:127084.

24. Majore I, Moretti P, Stahl F, Hass R, Kasper C. Growth and differentiation properties of mesenchymal stromal cell populations derived from whole human umbilical cord. Stem Cell Rev Rep. 2011;7(1):17-31.

25. Pereira RC, Costa-Pinto AR, Frias AM, Neves NM, Azevedo HS, Reis RL. In vitro chondrogenic commitment of human Wharton's jelly stem cells by coculture with human articular chondrocytes. J Tissue Eng Regen Med. 2017; 11(6):1876-87.

26. Zhang Y, Liu S, Guo W, Hao C, Wang M, Li X, Zhang X, Chen M, Wang Z, Sui X, Peng J, Wang Y, Lu S, Guo Q. Coculture of hWJMSCs and pACs in oriented scaffold enhances hyaline cartilage regeneration in vitro. Stem Cells Int. 2019;2019:5130152.

27. Zhang Y, Liu S, Guo W, Wang M, Hao C, Gao S, Zhang X, Li X, Chen M, Jing $X$, Wang Z, Peng J, Lu S, Guo Q. Human umbilical cord Wharton's jelly mesenchymal stem cells combined with an acellular cartilage extracellular matrix scaffold improve cartilage repair compared with microfracture in a caprine model. Osteoarthr Cartil. 2018;26(7):954-65.

28. Peterfy CG, Guermazi A, Zaim S, Tirman PF, Miaux Y, White D, Kothari M, Lu Y, Fye K, Zhao S, Genant HK. Whole-Organ Magnetic Resonance Imaging Score (WORMS) of the knee in osteoarthritis. Osteoarthritis Cartilage. 2004; 12(3):177-90.

29. Albright JC, Daoud AK. Microfracture and microfracture plus. Clin Sports Med. 2017;36(3):501-7

30. Mainil-Varlet P, Aigner T, Brittberg M, Bullough P, Hollander A, Hunziker E, Kandel R, Nehrer S, Pritzker K, Roberts S, Stauffer E. Histological assessment of cartilage repair: a report by the Histology Endpoint Committee of the International Cartilage Repair Society (ICRS). J Bone Joint Surg Am. 2003;2:45-57.

31. S. Liu, K.D. Hou, M. Yuan, J. Peng, L. Zhang, X. Sui, B. Zhao, W. Xu, A. Wang, S. Lu, Q. Guo, Characteristics of mesenchymal stem cells derived from Wharton's jelly of human umbilical cord and for fabrication of non-scaffold tissue-engineered cartilage, (1347-4421 (Electronic)).

32. Liu S, Yuan M, Hou K, Zhang L, Zheng X, Zhao B, Sui X, Xu W, Lu S, Guo Q. Immune characterization of mesenchymal stem cells in human umbilical cord Wharton's jelly and derived cartilage cells. Cell Immunol. 2012;278(1-2): $35-44$.

33. Liu S, Jia Y, Yuan M, Guo W, Huang J, Zhao B, Peng J, Xu W, Lu S, Guo Q. Repair of osteochondral defects using human umbilical cord Wharton's jelly-derived mesenchymal stem cells in a rabbit model. Biomed Res Int 2017;2017:8760383.

34. Kim YJ, Kim HJ, Im GI. PTHrP promotes chondrogenesis and suppresses hypertrophy from both bone marrow-derived and adipose tissue-derived MSCs. Biochem Biophys Res Commun. 2008;373(1):104-8.

35. Lindenmair A, Hatlapatka T, Kollwig G, Hennerbichler S, Gabriel C, Wolbank $\mathrm{S}$, Redl H, Kasper C. Mesenchymal stem or stromal cells from amnion and umbilical cord tissue and their potential for clinical applications. Cells. 2012; 1(4):1061-88.

36. Wang L, Ott L, Seshareddy K, Weiss ML, Detamore MS. Musculoskeletal tissue engineering with human umbilical cord mesenchymal stromal cells. Regen Med. 2011;6(1):95-109.

37. Ba K, Wei X, Ni D, Li N, Du T, Wang X, Pan W. Chondrocyte co-cultures with the stromal vascular fraction of adipose tissue in polyhydroxybutyrate/poly(hydroxybutyrate-co-hydroxyhexanoate) scaffolds: evaluation of cartilage repair in rabbit. Cell Transplant. 2019;28(11):1432-8.

38. Zheng P, Hu X, Lou Y, Tang K. A rabbit model of osteochondral regeneration using three-dimensional printed polycaprolactone-hydroxyapatite scaffolds coated with umbilical cord blood mesenchymal stem cells and chondrocytes. Med Sci Monitor Int Med J Exp Clin Res. 2019;25:7361-9.

39. Benya PD, Shaffer JD. Dedifferentiated chondrocytes reexpress the differentiated collagen phenotype when cultured in agarose gels. Cell. 1982; 30(1):215-24 
40. Darling EM, Athanasiou KA. Rapid phenotypic changes in passaged articular chondrocyte subpopulations. J Orthopaedic Res. 2005;23(2):425-32.

41. Filardo G, Perdisa F, Roffi A, Marcacci M, Kon E. Stem cells in articular cartilage regeneration. J Orthop Surg Res. 2016;11(1):42.

42. Inamdar SR, Knight DP, Terrill NJ, Karunaratne A, Cacho-Nerin F, Knight MM, Gupta HS. The secret life of collagen: temporal changes in nanoscale fibrillar pre-strain and molecular organization during physiological loading of cartilage. ACS Nano. 2017;11(10):9728-37.

43. Pfeiffer E, Vickers SM, Frank E, Grodzinsky AJ, Spector M. The effects of glycosaminoglycan content on the compressive modulus of cartilage engineered in type II collagen scaffolds. Osteoarthr Cartil. 2008;16(10):1237-44.

\section{Publisher's Note}

Springer Nature remains neutral with regard to jurisdictional claims in published maps and institutional affiliations.

Ready to submit your research? Choose BMC and benefit from:

- fast, convenient online submission

- thorough peer review by experienced researchers in your field

- rapid publication on acceptance

- support for research data, including large and complex data types

- gold Open Access which fosters wider collaboration and increased citations

- maximum visibility for your research: over $100 \mathrm{M}$ website views per year

At $\mathrm{BMC}$, research is always in progress.

Learn more biomedcentral.com/submissions 